\title{
Anti-osteoporosis effect of Notopterygium incisum Ting ex H. T. Chang extract in rats
}

\author{
Hai Ding*, Wenju Chang, Jian Yao, Fendou Liu, Qingliang Zeng, Zhenxing He \\ Department of Orthopedics, The First Affiliated Hospital of Bengbu Medical College, Bengbu, Anhui 233004, PR China \\ ${ }^{*}$ For correspondence: Email: dinghai133@126.com; Tel: +86-0552-3086163
}

Sent for review: 17 September 2018

Revised accepted: 16 September 2019

\begin{abstract}
Purpose: To investigate the effect of Notopterygium incisum Ting ex H.T. Chang extract (NICE) on ovariectomy-induced osteoporosis in rats.

Methods: Female Sprague-Dawley rats were randomly assigned to a normal group (control) and five ovariectomy (OVX) subgroups: OVX with vehicle (OVX), OVX with positive control drug alendronate sodium tablets (1.8 mg/kg/week), and OVX with varying NICE doses $(45,90$, or $180 \mathrm{mg} / \mathrm{kg} / \mathrm{day})$. After rats were subjected to ovariectomy for 4 weeks, alendronate or NICE were administered orally daily for 16 weeks. The bone mineral density (BMD) of the L4 vertebrae and right femurs of all rats was estimated. The serum hormones estradiol (E2), follicle-stimulating hormone (FSH) and luteinizing hormone (LH) levels and serum alkaline phosphatase (ALP), osteocalcin (OC) and telopeptides of collagen type I (CTX) levels of rats were detrmined.

Results: The results show that NICE dose-dependently inhibited bone mineral density (BMD) reduction of $L 4$ vertebrae and femurs $(p<0.05)$. Compared with OVX group, serum E2, FSH and LH levels was significantly increased in osteoporosis rats $(p<0.01)$, but serum ALP, CTX, and OC concentrations were significantly lower $(p<0.05)$. On the other hand, bone trabeculae in the three NICE groups and nilestriol group were all wider, while the space and connections increased.

Conclusion: These findings indicate that NICE mitigates OVX-induced osteoporosis in rats, and hence can potentially be developed for the management of osteoporosis.
\end{abstract}

Keywords: Notopterygium incisum, Osteoporosis, Ovariectomy, Bone tissue morphology

This is an Open Access article that uses a fund-ing model which does not charge readers or their institutions for access and distributed under the terms of the Creative Commons Attribution License (http://creativecommons.org/licenses/by/4.0) and the Budapest Open Access Initiative (http://www.budapestopenaccessinitiative.org/read), which permit unrestricted use, distribution, and reproduction in any medium, provided the original work is properly credited.

Tropical Journal of Pharmaceutical Research is indexed by Science Citation Index (SciSearch), Scopus, International Pharmaceutical Abstract, Chemical Abstracts, Embase, Index Copernicus, EBSCO, African Index Medicus, JournalSeek, Journal Citation Reports/Science Edition, Directory of Open Access Journals (DOAJ), African Journal Online, Bioline International, Open-J-Gate and Pharmacy Abstracts

\section{INTRODUCTION}

Postmenopausal osteoporosis is a type of systemic bone disease characterized by a reduction in bone density, degradation of bone microstructure and an alteration in serum markers of bone metabolism such as alkaline phosphatase (ALP), estradiol (E2) and interleukin-6 (IL-6) in postmenopausal females.
These results in bone fragility and an increased risk of fracture. Approximately one third of postmenopausal females suffer from osteoporosis usually due to marked reduction in estrogen levels [1].

According to data released by World Health Organization (WHO), osteoporosis affects approximately million people throughout Europe, 
USA, and Japan [2]. Incidence of osteoporosis increases dramatically with life expectancy. Accordingly, the risk of osteoporotic fractures and their associated costs rising rapidly due to aging populations [3]. In the elderly, hip fractures are closely associated with mortality [4]. Hormone deficiency is known to impair cancellous metaphyseal and reduce BMD in humans and animals. Therefore, estrogen deficiency in post-menopausal women has been regarded as a critical cause of this population susceptibility to osteoporosis [5]. Osteoporosis is twice as common in women as in men [6], and approximately one in three women over 50 years old experiences an osteoporotic fracture in her life time [7].

Clinically, hormone replacement therapy (HRT) has been a popular therapeutic strategy designed for postmenopausal osteoporosis $[8,9]$. However, long-term application of HRT has potential malignant effects on reproductive tissues [10-12]. Other medicines that stimulate bone formation (e.g., growth hormone, sodium uoride, and parathyroid hormone) or inhibit bone resorption (e.g., bisphosphonates and calcitonin) may prevent bone loss in established osteoporosis. However, these drugs are not effective for a large proportion of the population, especially in developing countries, and some drugs have side effects, such as gastrointestinal reactions, cancers, osteonecrosis of the jaw, and reduced skeletal strength $[13,14]$. Consequently, to substitute or reduce the medicines used currently, there are efforts to develop new drugs with improved therapeutic efficacy, fewer undesirable side effects, and lower cost.

Notopterygium incisum Ting ex H.T. Chang is a traditional Chinese medicate herb, which has been commonly used as an anti-aging agent, anti-osteoporosis and aphrodisiac in China [15]. This study investigated the therapeutic effects of NICE on ovariectomy-induced osteoporotic rats.

\section{EXPERIMENTAL}

\section{Preparation of NICE}

Herbal samples of Notopterygium incisum Ting ex H. T. Chang were collected from Nanning City, Guangxi Province in China in May 2017. Taxonomic identification of the plant was performed by Professor Ling Wang of College of Pharmacy, Bengbu medical college, in Bengbu of China. A voucher specimen (no. NICE 201705014) was deposited in the herbarium of College of Pharmacy, Bengbu Medical College, Bengbu, China for future reference.

\section{Animals and treatments}

Healthy six-month-old female Sprague-Dawley rats (weighing $220 \pm 20 \mathrm{~g}$ ) were provided by Anhui Province Experimental Animal center (certificate no. SYXK2006-0006). The animals had free access to feed and water, and were allowed to acclimatize for at least one week before use. The rat experiment was approved by Animal Care and Use Committee of the First Affiliated Hospital of Bengbu Medical College (approval ref no. 200805832) and carried out in compliance with the Directive 2010/63/EU on the handling of animals used for scientific purposes [16].

Sixty rats were randomly divided into six groups of ten rats each: normal control, OVX with vehicle (OVX), OVX with positive control drug Alendronate Sodium Tablets (ASD, 1.8 $\mathrm{mg} / \mathrm{kg} / \mathrm{week}$ ), and OVX with different NICE doses 82,164 or $328 \mathrm{mg} / \mathrm{kg} /$ day.

\section{BMD measurement}

The BMD of the L1-5 vertebrae and right femurs was estimated using dual-energy X-ray absorptiometry scanning (DEXA, GE Healthcare, USA). The measurements were expressed as mineral grams per $\mathrm{cm}^{2}$ of surface area. Scans were performed by the same blinded technician.

\section{Serum E2, FSH and LH levels in rats treated with NICE}

After the rats were sacrificed by cervical dislocation, rat serum was obtained by excising the eyeball. Serum E2, FSH and LH levels of rats were determined by ELISA method (Nanjing, Biological Engineering Research Institute Co. Ltd, Nanjing, China).

\section{Evaluation of serum bone markers in rats treated with NICE}

After the rats were sacrificed by cervical dislocation, rat serum alkaline phosphatase (ALP), osteocalcin (OC) and telopeptides of collagen type I (CTX) levels were determined by ELISA method (Nanjing Jiancheng Biological Engineering Research Institute Co. Ltd Nanjing, China).

\section{Statistical analysis}

The data are expressed as mean \pm SD. Statistical analysis was performed using one-way ANOVA combined with Bonferroni's multiple comparison test using SPSS 16.0. Differences 
were considered statistically significant at $p<$ 0.05 .

\section{RESULTS}

\section{BMD of L1-5 vertebrae and femurs}

The BMD of the L1-5 vertebrae and femurs is presented in Table 1. These results demonstrate that OVX significantly decreased BMD in the L4 vertebrae and femurs compared to the control group $(p<0.05)$. Compared to the OVX group, NICE treatment significantly increased the BMD in OVX L4 vertebrae and femurs $(p<0.05)$ in a dose-dependent manner. The positive control drug Fosamax also significantly increased the BMD of the L4 vertebrae and femurs (both $p<$ $0.05)$, which was similar to that observed in the H-NICE group $(p>0.05)$.

\section{Effect of NICE on serum E2, FSH and LH levels}

Compared to control group, serum E2, FSH and LH levels decreased significantly in OVX group rats $(p<0.01)$. Compared to the OVX group, NICE treatment significantly increased serum E2, FSH and LH levels $(p<0.05)$ in a dosedependent manner in rats (Table 2).

\section{Effect of NICE on serum ALP, CTx and OC concentrations}

Serum ALP, CTX, and OC concentrations in the OVX group were significantly higher when compared to the control group. After four weeks of treatment, the NICE $300 \mathrm{mg} / \mathrm{kg}$-treated group showed significantly lower serum ALP, CTx and OC concentrations when compared to the OVX group $(p<0.01)$ (Table 3$)$.

\section{DISCUSSION}

Postmenopausal osteoporosis is a systematic imbalance in which the speed of bone resorption is greater than the bone formation. This disease is caused by estrogen deficiency and results in microarchitectural changes, particularly bone remodeling.

Table 1: Effect of NICE on BMD of L4 vertebrae and femurs $(n=10)$

\begin{tabular}{lccc}
\hline Group & $\begin{array}{c}\text { Dose } \\
(\mathbf{m g} / \mathbf{k g})\end{array}$ & $\begin{array}{c}\text { BMD of vertebrum } \\
\left(\mathbf{g} / \mathbf{c m}^{2}\right)\end{array}$ & $\begin{array}{c}\text { BMD of femur } \\
\left(\mathbf{g} / \mathbf{c m}^{2}\right)\end{array}$ \\
\hline Control & - & $0.89 \pm 0.04$ & $0.82 \pm 0.03$ \\
OVX & - & $0.18 \pm 0.03$ & $0.15 \pm 0.04$ \\
ASD & 1.8 & $0.38 \pm 0.04^{*}$ & $0.42 \pm 0.03^{\star}$ \\
L-NICE & 45 & $0.30 \pm 0.02^{\star}$ & $0.27 \pm 0.04^{\star}$ \\
M-NICE & 90 & $0.41 \pm 0.03^{\star}$ & $0.23 \pm 0.04^{\star}$ \\
H-NICE & 180 & $0.76 \pm 0.03^{-}$ & $0.62 \pm 0.03$ \\
\hline
\end{tabular}

$P<0.05$ and " $p<0.01$ compared OVX group. L-NICE = low dose of NICE,

$\mathrm{M}-\mathrm{NICE}=$ middle dose of NICE, H-NICE = high dose of NICE, ASD: alendronate sodium tablets

Table 2: Effect of NICE on serum hormone levels $(n=10)$

\begin{tabular}{|c|c|c|c|c|}
\hline Group & Dose (mg/kg) & E2 (pmol/L) & FSH (IU/L) & LH (mlU/mL) \\
\hline Control & - & $9.32 \pm 0.11$ & $2.86 \pm 0.03$ & $3.81 \pm 0.09$ \\
\hline OVX & - & $1.58 \pm 0.10$ & $0.64 \pm 0.04$ & $1.42 \pm 0.06$ \\
\hline ASD & 1.8 & $4.54 \pm 0.12^{\pi \pi}$ & $1.92 \pm 0.05^{\star}$ & $2.44 \pm 0.03^{x}$ \\
\hline L-NICE & 45 & $2.63 \pm 0.18$ & $0.86 \pm 0.04$ & $1.96 \pm 0.11$ \\
\hline M-NICE & 90 & $3.91 \pm 0.19$ & $1.70 \pm 0.03$ & $2.52 \pm 0.08$ \\
\hline H-NICE & 180 & $4.72 \pm 0.13^{\prime \prime}$ & $1.91 \pm 0.04$ & $2.98 \pm 0.06$ \\
\hline
\end{tabular}

$P<0.05$ and " $p<0.01$ versus OVX group. L-NICE: low dose of NICE, M-NICE: middle dose of NICE, H-NICE: high dose of NICE, ASD: alendronate sodium tablets

Table 3: Effect of NICE on serum ALP, CTx, and OC levels $(n=10)$

\begin{tabular}{|c|c|c|c|c|}
\hline Group & Dose $(\mathrm{mg} / \mathrm{kg})$ & ALP (U/L) & CTX (ng/mL) & OC (ng/mL) \\
\hline Control & - & $135.3 \pm 3.0$ & $21.8 \pm 1.5$ & $34.5 \pm 1.6$ \\
\hline OVX & - & $272.5 \pm 5.1^{\star \pi}$ & $87.4 \pm 1.1^{\star \pi}$ & $86.1 \pm 2.2^{\pi x}$ \\
\hline ASD & 1.8 & $178.7 \pm 4.5$ & $52.4 \pm 2.4$ & $58.6 \pm 1.5^{n}$ \\
\hline L-NICE & 45 & $223.2 \pm 5.1$ & $76.9 \pm 2.0$ & $79.1 \pm 1.6$ \\
\hline M-NICE & 90 & $168.7 \pm 4.0$ & $66.7 \pm 1.4$ & $74.8 \pm 1.5$ \\
\hline H-NICE & 180 & $154.3 \pm 3.4^{\pi \times}$ & $55.2 \pm 1.6^{\pi \pi}$ & $63.7 \pm 1.1^{\pi \pi}$ \\
\hline
\end{tabular}

"P<0.05 and " $p<0.01$ versus OVX group. L-NICE: low dose of NICE, M-NICE: middle dose of NICE, H-NICE: high dose of NICE, ASD: Alendronate Sodium Tablets 
Certain molecules co-ordinate the actions of osteoblasts and osteoclasts during bone remodeling. Therefore, new anti-osteoporosis drugs is urgently needed. In recent years, Chinese medicinal herbal extracts have been extensively investigated for their pharmacological effects related to bone protection [17].

Decreased BMD is one of the major factors that jeopardize bone strength, resulting in increased susceptibility to fractures. Thus, BMD measurement can best predict fracture risk [18]. The results presented showed that OVX reduced $B M D$ in the right femurs and L4 vertebrae which are rich in trabecular bone, while treatment with NICE dose-dependently prevented the decreases in BMD. Although BMD is among the strongest predictors of facture resistance, empirical observations and theoretical analyses show that the biomechanical properties of bone and trabecular microarchitecture influence trabecular bone strength as well [19].

Estradiol plays an important role in sclerotin of humans. When female serum estradiol level decreases significantly, osteoporosis will occur [20]. Compared to the OVX group, NICE treatment significantly increased serum E2, FSH and $\mathrm{LH}$ levels in a dose-dependent manner in rats. In the experiments, OVX resulted in significant decrease in femur BMD after four weeks. The BMD loss was accompanied by a significant increase in bone remodeling, as evidenced by the increased biochemical bone turnover markers such as serum ALP, CTx, and OC levels [21].

In the present study, oral administration of NICE at the dose $328 \mathrm{mg} / \mathrm{kg}$ significantly decreased BMD loss, which was accompanied by the decrease in serum ALP, CTX, and OC levels compared to a OVX control group. These results suggest that Bushen Jiangu Decoction could decreases bone loss by inhibiting bone turnover induced by OVX.

\section{CONCLUSION}

The findings of this study indicate that NICE prevents OVX-induced osteoporosis in rats, and therefore can potentially be used for the treatment of postmenopausal osteoporosis in elderly women.

\section{DECLARATIONS}

\section{Conflict of interest}

No conflict of interest is associated with this work.

\section{Contribution of authors}

We declare that this work was done by the authors named in this article and all liabilities pertaining to claims relating to the content of this article will be borne by the authors. Hai Ding designed all the experiment and revised the manuscript. Wenju Chang, Jian Yao and Fendou Liu performed the experiments, Qingliang Zeng and Zhenxing He wrote the manuscript.

\section{Open Access}

This is an Open Access article that uses a funding model which does not charge readers or their institutions for access and distributed under the terms of the Creative Commons Attribution License (http://creativecommons.org/licenses/by/ 4.0) and the Budapest Open Access Initiative (http://www.budapestopenaccessinitiative.org/rea d), which permit unrestricted use, distribution, and reproduction in any medium, provided the original work is properly credited.

\section{REFERENCES}

1. NIH Consensus Development Panel on Osteoporosis Prevention, Diagnosis, and therapy. Osteoporosis prevention, diagnosis, and therapy. J Am Med Ass 2001; 285: 785-795.

2. Eastell R, Black DM, Boonen S. Effect of once-yearly zoledronic acid five milligrams on fracture risk and change in femoral neck bone mineral density. J Clin Endo Metabol 2009; 94: 3215-3225.

3. Burge $R$, Dawson-Hughes $B$, Solomon $D H$. Incidence and economic burden of osteoporosis-related fractures in the United States 2005-2025. J Bone Min Res 2007; 22: 465-475.

4. Omsland TK, Emaus N, Tell GS. Mortality following the first hip fracture in Norwegian women and men. A NOREPOS study. Bone 2014; 63: 81-86.

5. Marcus R. An expanded overview of postmenopausal osteoporosis. J Mus Neuronal Int 2002; 2: 195-197.

6. Sugerman DT. Osteoporosis JAMA 2014; 311: 104-105.

7. Johnell O, Kanis JA. An estimate of the worldwide prevalence and disability associated with osteoporotic fractures. Osteoporosis Inter 2006; 17: 1726-1733.

8. Stevenson JC. Justification for the use of HRT in the long-term prevention of osteoporosis. Maturitas 2005; 51: 113-126.

9. Prelevic GM, Kocjan T, Markou A. Hormone replacement therapy in postmenopausal women. Minerva Endocrinologica 2015; 30: 27-36.

10. Gray S. Breast cancer and hormone-replacement therapy: the Million Women Study. Lancet 2013; 362: 1332-1333.

11. Orija IB, Mehta A. Hormone replacement therapy: current controversies. Clin Endocrinol 2013; 59: 657-658.

Trop J Pharm Res, October 2019; 18(10):2054 
12. Lacey JV, Mink PJ, Lubin JH. Menopausal hormone replacement therapy and risk of ovarian cancer. J Ame Med Asso 2012; 288: 334-341.

13. Rossouw JE, Anderson GL, Prentice RL. Risks and benefits of estrogen plus progestin in healthy postmenopausal women: principal results from the Women's Health Initiative randomized controlled trial. J Ame Med Assoc 2012; 288: 321-333.

14. Lee JK, Kim KW, Choi JY. Bisphosphonates-related osteonecrosis of the jaw in Korea: a preliminary report. $J$ Korean Asso of Oral and Maxi Surgeons 2013; 39: 9-13.

15. Donnapee S, Li J, Yang X. Cuscuta chinensis Lam.: A systematic review on ethnopharmacology, phytochemistry and pharmacology of an important traditional herbal medicine. J Ethnopharmacol 2014; 18 : 292-308.

16. European Commission. Directive 2010/63/EU on the protection of animals used for scientific purposes [cited 2013 Jan 16]. Available from: http://ec.europa.eu/environment/chemicals/lab_animals/l egislation_en.htm.

17. Wronski TJ, Dann LM, Scott KS. Endocrine and pharmacological suppressors of bone turnover protect against osteopenia in ovariectomized rats. Endoc 2009; 125: 810-816.

18. Turner RT, Vandersteenhoven JJ, Bell NH. The effects of ovariectomy and 17 beta-estradiol on cortical bone histomorphometry in growing rats. J Bone Min Res 2010; 2: 115-122.

19. Dai $S Q$, Yu LP, Shi $X$, Wu H, Shao P, Yin GY. Serotonin regulates osteoblast proliferation and function in vitro. Braz J Med Biol Res 2014; 47: 759-765.

20. Dallas SL, Prideaux M, Bonewald LF. The osteocyte: an endocrine cell and more. Endocr Rev 2013; 34: 658690.

21. Carson JA, Baltgalvis KA. Interleukin 6 as a key regulator of muscle mass during cachexia. Exer Sport Sci Rev 2010; 38: 168-176. 